Submitted to the Editor of the Astrophysical Journal Letters

\title{
Accretion Processes in the Nucleus of M31
}

\author{
Siming Liu $^{1}$ and Fulvio Melia ${ }^{1,2,3}$ \\ ${ }^{1}$ Physics Department, The University of Arizona, Tucson, AZ 85721 \\ ${ }^{2}$ Steward Observatory, The University of Arizona, Tucson, AZ 85721
}

Received __ ; accepted _

\footnotetext{
${ }^{3}$ Sir Thomas Lyle Fellow and Miegunyah Fellow.
} 


\begin{abstract}
The hypothesized supermassive black hole in the nucleus of M31 (which we shall hereafter call M31*) has many features in common with Sgr A* at the Galactic Center, yet they differ in several significant and important ways. Though M31* is probably ten times heavier, its radio luminosity at $3.6 \mathrm{~cm}$ is only one third that of $\mathrm{Sgr} \mathrm{A}^{*}$. At the same time, M31* is apparently thousands of times more luminous in X-rays than its Galactic Center counterpart. Thus, a comparative study of these objects can be valuable in helping us to understand the underlying physical basis for their activity. We show here that the accretion model being developed for Sgr $A^{*}$ comprises two branches of solutions, distinguished by the relative importance of cooling compared to compressional heating at the radius $r_{C}$ where the ambient gas is captured by the black hole. For typical conditions in the ISM, the initial temperature $\left(T\left[r_{C}\right] \sim 10^{6}-10^{7} \mathrm{~K}\right)$ sits on the unstable branch of the cooling function. Depending on the actual value of $T\left(r_{C}\right)$ and the accretion rate, the plasma settles either onto a hot branch (attaining a temperature as high as $10^{10} \mathrm{~K}$ or so at small radii) or a cold branch, in which $T$ drops to $\sim 10^{4} \mathrm{~K}$. Sgr A* is presumably a 'hot' black hole. We show here that the VLA, UV and Chandra observations of M31* reveal it to be a member of the 'cold' black hole family. We discuss several predicted features in the spectrum of M31* that may be testable by future multi-wavelength observations, including the presence of a prominent UV spike (from hydrogen line emission) that would be absent on the hot branch.
\end{abstract}

Subject headings: accretion-black hole physics - Galaxy: center-galaxies: individual (M31) - galaxies: nuclei-X-rays: galaxies 


\section{Introduction}

The discovery of an unresolved radio point source (labeled as M31*) in the nucleus of M31 (Crane, Dickel \& Cowan 1992) led to an early suggestion (Melia 1992b) that its nature may be similar to that of the supermassive black hole candidate, Sgr A*, at the center of our own Galaxy. The distance to M31 renders its nuclear environment difficult to observe with anything approaching the spatial resolution now available for the Galactic Center, but certain cues suggest that in both cases we may be dealing with a massive pointlike object accreting gas from the interstellar medium, possibly from stellar winds. However, there do exist several notable differences between the two systems, making a comparative analysis of their emitting regions highly desirable.

The Hubble Space Telescope (HST) resolved the nucleus of M31 into two components (P1 and P2) separated by $0.5 \approx 1.9 \mathrm{pc}$ (Lauer et al. 1993). The rotational velocity measurements of the stars near the nucleus (Kormendy \& Bender 1999) suggest that this structure is a torus of stars orbiting a central dark, object of mass $3.0 \times 10^{7} M_{\odot}$ in a slightly eccentric trajectory (Tremaine 1995). High resolution UV images of the nucleus show that there is a group of UV-bright stars between P1 and P2 (Brown et al. 1998), making it very difficult to study the compact object at this frequency. A pre-Costar HST far-UV observation in 1991 (King, Stanford \& Crane 1995) could not resolve this cluster from P2, but it did show that the region near P2 has a UV upturn that is much stronger than P1 and the surrounding bulge light. These authors argue that the FUV flux level $(\sim 3 \mu \mathrm{Jy})$ from $\mathrm{P} 2$ is not from stars, but is rather a high-frequency extension of the radio source M31*. This view is supported by the arguments of Kormendy and Bender (1999) that the central super-massive black hole probably lies at the center of this UV-bright cluster.

In the radio, there now exist measurements of $\mathrm{M}^{*}{ }^{*}$ 's flux at two epochs (Crane et al. 1992; Crane et al. 1993), showing that, at least at $3.6 \mathrm{~cm}$, its luminosity is variable on a time scale of a year or less. But even though M31* is apparently ten times more massive than the black hole at the Galactic Center, its power at $3.6 \mathrm{~cm}$ is only one third that of $\mathrm{Sgr} \mathrm{A}^{*}$ (assuming a distance to M31 of 784 kpc; Stanek \& Garnavich 1998). This contrasts with the situation at higher energy, where M31* is thousands of times more luminous than the latter in X-rays (Garcia et al. 2000). 
The recent Chandra observation also shows that M31* has a soft X-ray spectrum, while the X-ray spectrum of Sgr A* may be harder (Baganoff et al. 2001; Melia, Liu, \& Coker 2000b).

The recent modeling of Sgr A* (Melia, Liu \& Coker 2000a, 2000b), taking into account the latest high precision $\mathrm{mm}$, sub-mm and Chandra measurements, suggests that (at least in this source) the rate of accretion $(\dot{M})$ toward small radii $\left(<30 r_{S}\right.$, where $r_{S}$ is the Schwarzschild radius $\left.\equiv 2 G M / c^{2}\right)$ must be significantly smaller than the rate of wind capture at the Bondi-Hoyle radius $r_{C} \equiv 2 G M / v_{\infty}^{2}$, where $v_{\infty}$ is the velocity of the ambient gas flowing past the central object. Hydrodynamic simulations of the environment surrounding Sgr A* (Coker \& Melia 1997) indicate that the infalling gas circularizes when it approaches within $5-25 r_{S}$ of the black hole. The sub-mm "excess" emission seen in the spectrum of this source appears to be associated with radiation produced within the inner Keplerian region. This view is supported by the linear polarization observed in Sgr $\mathrm{A}^{*}$ at $\mathrm{mm}$ and sub-mm wavelengths (Aitken et al. 2000), which is difficult to reconcile with other geometries of the emitting region. Both the degree of polarization and the rotation (by about $90^{\circ}$ ) in the position angle appear to be consistent with a transition of the emitting region within the Keplerian flow from optically thick emission (at mm and longer wavelengths) to optically thin emission in the sub-mm range (Melia 1992a; 1994). The overall spectrum from this region includes a high-energy component due to bremsstrahlung and inverse Compton scattering processes, which may account for the Chandra X-ray source coincident with Sgr A* if it turns out to be the actual counterpart to this object (Baganoff et al. 2001).

The situation in the nucleus of M31 may be quite different for several reasons. First, $v_{\infty}$ around Sgr A* may be anomalously high due to the presence of strong wind-producing young stars (e.g., Najarro et al. 1997). In M31, on the other hand, very little is known about the nuclear gas motion, although on a larger scale, Rubin \& Ford (1971) find that the plasma within the inner 400 pc region shows a complex velocity field superposed on the rapid rotation, with evidence for expansion at velocities up to $\sim 100 \mathrm{~km} \mathrm{~s}^{-1}$. Second, the central stellar distribution, as noted above, is clearly anisotropic, with P2 lying a mere $0^{\prime \prime} 1\left(\approx 1.3 \times 10^{5} r_{S}\right)$ away from the central black hole (Kormendy \& Bender 1999). In M31, a lower $v_{\infty}$, together with the disruption to the gas 
flow caused by these stars, may result in a greater $\dot{M}$ with a smaller specific angular momentum.

It is well known that the gas temperature $T$ near $r_{C}$ depends on the kinetic energy flux brought into this region by the pre-shocked gas (see Coker \& Melia 2000). An important consequence of a weaker flow is a value of $T$ as low as $10^{6} \mathrm{~K}$ at large radii, compared with $\sim 10^{7}$ $\mathrm{K}$ in the Galactic Center. A thin plasma under these conditions sits on the unstable branch of the cooling function (Gehrels \& Williams 1993). As it continues to fall toward the accretor, it can either heat up to $>10^{10} \mathrm{~K}$, at which point the cooling rate balances heating, or it cools down to $\sim 10^{4} \mathrm{~K}$, where the energy loss rate drops precipitously. In general, a lower initial temperature and a larger particle number density force the plasma into the lower $T$ stable branch while the higher $T$ branch is favored by the gas starting its descent with a high $T$ and low density.

In this Letter, we explore this dichotomy in accretion profiles, and suggest that Sgr A* represents the hot branch, while M31* is a member of the cold branch family of accreting black holes. In both cases, the initial temperature near $r_{C}$ falls within the unstable region, but M31* is accreting at a higher rate than Sgr $\mathrm{A}^{*}$. If indeed the plasma in M31* is stabilized at $\sim 1.5 \times 10^{4}$ K, its FUV spectrum should be characterized by strong $\mathrm{H}$ line emission, while the soft X-rays are produced by recombination in the hot outer region. This is in contrast with Sgr $\mathrm{A}^{*}$, where the X-rays are produced by the inner hot gas. In addition, we inferred a mass loss for Sgr $\mathrm{A}^{*}$ to account for the variable $\dot{M}$ toward small radii. This may not occur in M31* where the inflowing gas is much colder and hence more tightly bound (see Fig. 1). We emphasize that our analysis and conclusions regarding M31* are based on the assumption that both the $3.6 \mathrm{~cm}$ and Chandra measurements represent the actual source strength, rather than upper limits. One of our goals is to make available several testable predictions for the next generation of coordinated observations.

\section{Accretion from the Ambient Medium and Calculation of the Spectrum}

The currently available spectral measurements for the nuclear region in M31 are listed in the Table. The column labeled "Integration Area" shows the region over which the intensity was 
integrated in order to arrive at the flux measurement quoted in column 3. The angular resolution for the $3.6 \mathrm{~cm}$ observations was $00^{\prime \prime} 42 \times 0^{\prime \prime} .31$ and $0^{\prime \prime} .29 \times 0^{\prime \prime} \cdot 26$, respectively, which is sufficient to attribute the observed flux to a single point source. The FOC (on $H S T$ ) observations of M31* are hampered by contamination from the blue stars around the center (Brown et al. 1998), so these should be considered as upper limits. However, the $0.175 \mu \mathrm{m}$ point represents the flux from this region once the contribution from these stars has been subtracted out (King et al. 1995). With a spatial resolution of $0 !$, Chandra was able to resolve the nucleus of M31 into five point sources (Garcia et al. 2000). These authors infer that the X-ray counterpart to M31* is strongly variable, when the recent data are compared to earlier Einstein observations of the TF 56 source (Trinchieri \& Fabbiano 1991).

A direct application of the Sgr A* accretion model (e.g., Coker \& Melia 2000; Melia, Liu \& Coker 2000b) to M31* immediately runs into problems because of the large disparity between the radio to X-ray flux ratios of these two sources. The spectrum of M31* simply does not support the idea that the inflowing gas is hot $\left(\sim 10^{10} \mathrm{~K}\right.$ or so) at small radii. We have therefore explored the characteristics of the accretion model in the high- $\dot{M}$ context to gauge the importance of rapid cooling in this environment.

The relativistic black hole accretion model was pioneered by Shapiro (1973), and later developed by several workers, the more recent of whom applied this picture to Sgr A* (e.g., Melia 1992a, 1994). Our calculation of the accretion profile for M31* follows the prescription of Coker \& Melia (2000), though with several important differences. First, we will restrict our attention to the cold branch solutions for which the impact of a magnetic field $B$ is negligible. This assumes that $B$ is sub-equipartition compared to the thermal energy density, following the arguments of Kowalenko \& Melia (1999), and the application to Sgr A* in the outer region (Coker \& Melia 2000). (Here the magnetic pressure never exceeds $\sim 2 \times 10^{-2}$ times the thermal pressure.) Thus, it is not important here to worry about an empirical fit for $B$, as we did for Sgr A*. Second, we are in a domain where cooling dominates the energy equation, especially at large radii where the conditions warrant a careful treatment of line cooling (Gehrels \& Williams 1993). Thus, whereas 
this effect could be ignored in the case of Sgr $\mathrm{A}^{*}$, we must include it here, and for this we adopt the cooling function used by these authors, corresponding to a thin gas with cosmic abundances.

\begin{tabular}{|c|c|c|c|c|c|c|}
\hline $\begin{array}{c}\lambda \\
\text { or Energy band }\end{array}$ & $\begin{array}{c}\nu \\
(\mathrm{Hz})\end{array}$ & $\begin{array}{c}F_{\nu} \\
(\mathrm{Jy})\end{array}$ & $\begin{array}{c}\text { Integration } \\
\text { Area }\end{array}$ & $\begin{array}{c}\text { Telescope } \\
\text { or Instrument }\end{array}$ & Date & Notes \\
\hline $3.6 \mathrm{~cm}$ & $8.4 \times 10^{9}$ & $(28 \pm 5) \times 10^{-6}$ & $0^{\prime \prime} 42 \times 00^{\prime \prime} .31$ & VLA & June 1990 & 1 \\
\hline $3.6 \mathrm{~cm}$ & $8.4 \times 10^{9}$ & $(39 \pm 5) \times 10^{-6}$ & $0^{\prime \prime} .29 \times 0^{\prime \prime} .26$ & VLA & Nov 1992 & 2 \\
\hline $100 \mu \mathrm{m}$ & $3.0 \times 10^{12}$ & 12.0 & $2{ }^{\prime} 0 \times 22^{\prime} 0$ & IRAS & 1983 & 3,4 \\
\hline $60 \mu \mathrm{m}$ & $5.0 \times 10^{12}$ & 7.1 & $2 ! 0 \times 2.0$ & IRAS & 1983 & 3,4 \\
\hline $25 \mu \mathrm{m}$ & $1.2 \times 10^{13}$ & 0.91 & $2^{\prime} .0 \times 22^{\prime} 0$ & IRAS & 1983 & 3,4 \\
\hline $12 \mu \mathrm{m}$ & $2.5 \times 10^{13}$ & 1.83 & $2^{\prime} .0 \times 2^{\prime} .0$ & IRAS & 1983 & 3,4 \\
\hline $0.75 \mu \mathrm{m}$ & $4.0 \times 10^{14}$ & 0.746 & $190^{\prime} \times 60^{\prime}$ & $0.9 \mathrm{~m}(\mathrm{KPNO})$ & Sep 1991 & 5 \\
\hline $0.61 \mu \mathrm{m}$ & $5.0 \times 10^{14}$ & 0.417 & $190^{\prime} \times 60^{\prime}$ & $0.9 \mathrm{~m}(\mathrm{KPNO})$ & Sep 1991 & 5 \\
\hline $0.53 \mu \mathrm{m}$ & $5.6 \times 10^{14}$ & 0.278 & $190^{\prime} \times 60^{\prime}$ & $0.9 \mathrm{~m}(\mathrm{KPNO})$ & Sep 1991 & 5 \\
\hline $0.46 \mu \mathrm{m}$ & $6.5 \times 10^{14}$ & 0.177 & $190^{\prime} \times 60^{\prime}$ & $0.9 \mathrm{~m}(\mathrm{KPNO})$ & Sep 1991 & 5 \\
\hline $0.40 \mu \mathrm{m}$ & $7.5 \times 10^{14}$ & 0.075 & $190^{\prime} \times 60^{\prime}$ & $0.9 \mathrm{~m}(\mathrm{KPNO})$ & Sep 1991 & 5 \\
\hline $0.35 \mu \mathrm{m}$ & $8.6 \times 10^{14}$ & 0.024 & $190^{\prime} \times 60^{\prime}$ & $0.9 \mathrm{~m}(\mathrm{KPNO})$ & Sep 1991 & 5 \\
\hline $0.280 \mu \mathrm{m}$ & $1.1 \times 10^{15}$ & $4.85 \times 10^{-4}$ & $2^{\prime \prime} 8 \times 2^{\prime \prime} .8$ & FOC on HST & Feb 1994 & 6 \\
\hline $0.199 \mu \mathrm{m}$ & $1.5 \times 10^{15}$ & $2.47 \times 10^{-4}$ & $2^{\prime \prime} .8 \times 2^{\prime \prime} .8$ & FOC on HST & Feb 1994 & 6 \\
\hline $0.175 \mu \mathrm{m}$ & $1.7 \times 10^{15}$ & $6.7 \times 10^{-6}$ & $0^{\prime \prime} 5 \times 0^{\prime \prime} .5$ & FOC on HST & 1991 & 7 \\
\hline $0.3-1.5 \mathrm{keV}$ & $(0.7-3.6) \times 10^{17}$ & $1.8 \times 10^{-7}$ & $2^{\prime \prime} .0 \times 2^{\prime \prime} .0$ & Chandra & Oct 1999 & 8 \\
\hline $1.5-7.0 \mathrm{keV}$ & $(0.4-1.7) \times 10^{18}$ & $7.8 \times 10^{-10}$ & $2^{\prime \prime} .0 \times 2^{\prime \prime} .0$ & Chandra & Oct 1999 & 8 \\
\hline
\end{tabular}

Notes: (1) Crane, Dickel \& Cowan. 1992. (2) Crane et al. 1993. (3) Soifer et al. 1986. (4) Neugebauer et al. 1984. (5) Mcquade, Calzetti \& Kinney 1995. (6) Brown et al. 1998. (7) King, Stanford \& Crane. 1995. (8) Garcia et al. 2000.

Without a specific determination of the gas conditions within a parsec or so of M31*, we are compelled to treat the gas kinematics as an unknown. We therefore adopt a fiducial wind velocity $v_{\infty}$ of $500 \mathrm{~km} \mathrm{~s}^{-1}$. In the cold branch solutions, a lower value of $v_{\infty}$ simply cools the 
gas faster because $\dot{M}$ is higher. The corresponding Bondi-Hoyle capture radius for M31* is then $r_{C}=3.6 \times 10^{5} r_{S}(\approx 0$ ' 28$)$, which by the way, is larger than the distance from M31* to P2 (see above). With $M$ and $v_{\infty}$ known, the accretion rate then depends on the gas density, which unfortunately is also poorly constrained. However, Ciardullo et al. (1988) argue that the ionized-gas density gradient in the nucleus of M31 is dramatic, dropping from $n_{e} \sim 10^{4}$ electrons $\mathrm{cm}^{-3}$ at a radius of $\sim 7^{\prime \prime}$ to $10^{2}$ electrons $\mathrm{cm}^{-3}$ at $\sim 1^{\prime}$. Thus, simply putting $\dot{M} \sim \pi r_{C}^{2} m_{p} n_{e} v_{\infty}$, where $m_{p}$ is the proton mass, and $n_{e}=10^{4} \mathrm{~cm}^{-3}$, we see that $\dot{M}$ may be as high as $10^{25} \mathrm{~g} \mathrm{~s}^{-1}$.

The temperature at the outer boundary $\left(\sim r_{C}\right)$ is estimated by assuming that the kinetic energy in the gas is thermalized by shocks, for which $6 R_{g} T\left(r_{C}\right) \sim\left(3 v_{\infty} / 4\right)^{2}$, where $R_{g}$ is the gas constant and we have assumed a mean molecular weight per particle of $1 / 2$. The principal radiating mechanisms are radiative recombination, and thermal bremsstrahlung emission. Electron-positron pair production, cyclo/synchrotron radiation and Comptonization are all negligible due to the low temperature and sub-equipartition magnetic field in the cold branch solutions. For simplicity, we here consider only $\mathrm{H}$ recombination to calculate the line emission. A more sophisticated treatment that includes the contribution from all the ions will be discussed elsewhere. For this, the ionization fraction may be calculated according to the prescription given in Rossi et al. (1997). At the prevalent temperature $\left(\sim 1.4 \times 10^{4} \mathrm{~K}\right)$ in the cold region, ignoring the other line emissivities amounts to an error of at most $\sim 15 \%$ (Brown \& Mathews 1970).

\section{Results and Conclusions}

The principal distinguishing feature between the hot and cold branch solutions is illustrated in Figure 1, which shows the temperature profile of the accreting gas as a function of radius. The emphasis here is the dependence of this profile on the initial temperature, so all the other physical conditions are identical for the three cases included on this plot, and are based on the best fit model for the spectrum of M31* (dark, solid curve). In all cases, the outer boundary is taken to be $r_{0}=10^{5} r_{S}\left(\approx 0^{\prime \prime} .08\right)$, with an accretion rate $\dot{M}=1.5 \times 10^{24} \mathrm{~g} \mathrm{~s}^{-1}$. The ratio of thermal to gravitational energy density at $r_{0}$, which characterizes the initial temperature, is $0.19,0.17$ and 
0.15, respectively, for the thin, solid curve (the sole hot branch solution), the thick, solid curve (the best fit model), and the dotted curve. With an appreciably lower $\dot{M}$, Sgr A* lies comfortably within the category of hot branch solutions since its initial temperature appears to be larger than that of M31* (at $\sim 3 \times 10^{6} \mathrm{~K}$ ). For the latter, however, the gas cools quickly as it collapses towards smaller radii, and has reached the cold, stable branch of the cooling curve $\left(\right.$ at $\left.\sim 10^{4} \mathrm{~K}\right)$ by the time it crosses $\sim 10^{3} r_{S}$. Thereafter, the heating due to compression never quite catches up and the gas continues to radiate efficiently as it plummets toward the event horizon.

The spectrum produced by this plasma is shown in Figure 2 (dark, solid curve), in which we have also indicated the contribution (mostly due to bremsstrahlung emission) from the hot $\left(T>10^{6} \mathrm{~K}\right)$, outer region (thin, solid curve), and the cooler $\left(T<10^{6} \mathrm{~K}\right)$, inner zone (dotted curve). The transition radius between these two domains is approximately $10^{3} r_{S}$ (see Fig. 1). The most constraining data on this plot are the VLA measurements (Crane et al. 1992; Crane et al. 1993), the FUV point inferred from the subtraction of starlight (King et al. 1995), and the more recent Chandra results (shown as a butterfly below $1.5 \mathrm{keV}$ and as an upper limit above this energy; Garcia et al. 2000). We see that the X-rays are produced in the outer, hot region, whereas the FUV spike arises from hydrogen line emission, predominantly in the cooler, inner zone. The radio portion of the spectrum is produced throughout the accretion volume.

We would thus expect M31*'s X-ray flux to vary on a time scale $\tau \sim 10^{3} r_{S} / v_{f f} \leq 4$ months, where the free-fall velocity $v_{f f}$ at $10^{3} r_{S}$ is approximately $c / 30$. Its radio flux, which is produced throughout the volume of captured gas, should also vary on a time scale no larger than this, though only a portion of this variability is expected to be correlated with the X-rays. Since some of the radio emission occurs at smaller radii, we expect that relative to the $\mathrm{X}$-rays, the $3.6 \mathrm{~cm}$ emission ought to display variability with a broader range of time scales.

Figure 2 also highlights one of the main features that distinguish the spectra of the hot and cold solutions - the FUV spike due to line emission, which is absent in the former, but is very prominent in the latter. This may be the origin of the peculiar UV upturn near P2 noted by King et al. (1995) in their analysis of the non-stellar contribution to the UV flux. The strength of this 
spectral component suggests that additional UV observations of the region near P2 are called for. The variability in the UV flux appears to be correlated with both the flux at $3.6 \mathrm{~cm}$, and the $\mathrm{X}$-rays, though the tightest correlation is expected to occur between the latter two.

In recent years, several other models have been invoked to account for the emissivity of supermassive black holes in the cores of nearby galaxies, including Sgr A*. ADAF (Narayan, Yi, \& Mahadevan 1995) and ADIOS (Blandford \& Begelman 1999) disk models differ from the model we have described here in several ways, including (1) the accreting gas has considerably more angular momentum than we have invoked, and (2) the gas separates into a two-temperature plasma, in which the protons become very hot (analogous to our hot-branch solution). However, the VLA point appears to rule out this possibility in the case of $\mathrm{M} 31^{*}$, for the simple reason that an optically thick emitter at this temperature, with a scale size corresponding to $M=3 \times 10^{7} M_{\odot}$, would simply produce too much flux at $3.6 \mathrm{~cm}$. In addition, a hot, optically-thin emitter does not produce the correct X-ray spectrum. These constraints also seem to argue against a jet model (Falcke \& Markoff 2000), since the emitting particles in this picture would have physical characteristics similar to those of our hot-branch solution. The combination of radio and X-ray measurements constitutes a powerful diagnostic. Future co-ordinated multi-wavelength observations of this intriguing supermassive black hole candidate would be invaluable.

We are indebted to Fred Baganoff, Mike Garcia, Ivan King, and Mark Morris for very valuable discussions and clarifications. This work was supported by a Sir Thomas Lyle Fellowship and a Miegunyah Fellowship in Melbourne, and by NASA grants NAG5-8239 and NAG5-9205. 


\section{REFERENCES}

Aitken, D.K., Greaves, J., Chrysostomou, A., Jenness, T., Holland, W., Hough, J.H., Pierce-Price, D. \& Richer, J. 2000, ApJ Letters, 534, L173

Baganoff, F. et al. 2001, ApJ submitted

Blandford, R.D. \& Begelman, M.C. 1999, MNRAS, 303, L1

Brown, T.M., Ferguson, H.C., Stanford, S.A. \& Deharveng, J.-M. 1998, ApJ, 504, 113

Brown, R.L. \& Mathews, W.G., 1970, ApJ, 160, 939

Ciardullo, R., Rubin, V.C., Jacoby, G.H., Ford, H.C. \& Ford, W.K. Jr. 1988, AJ, 95, 438

Coker, R.F. \& Melia, F. 1997, ApJ Letters, 488, L149

Coker, R.F. \& Melia, F. 2000, ApJ, 534, 723

Crane, P.C., Dickel, J.R. \& Cowan, J.J. 1992, ApJ Letters, 390, L9

Crane, P.C., Cowan, J.J., Dickel, J.R. \& Roberts, D.A. 1993, ApJ Letters, 417, L61

Falcke, H., Goss, W.M., Matsuo, H., Teuben, P., Zhao, J.-H. \& Zylka, R. 1998, ApJ, 499, 731

Falcke, H. \& Markoff, S. 2000, A\&A, in press

Garcia, M.R., Murray, S.S., Primini, F.A., Forman, W.R., McClintock, J.E. \& Jones, C. 2000, ApJ Letters, 537, L23

Garcia, M.R., Murray, S.S., Primini, F.A., Forman, W.R., Jones, C., \& McClintock, J.E. 2001, IAU 205, Galaxies at the Highest Angular Resolution, eds. Schilizzi, Vogel, Paresce, \& Elvis, in press.

Gehrels, N. \& Williams, E.D. 1993, APJ Letters, 418, 25.

Hjellming, R.M. \& Smarr, L.L. 1982, ApJ Letters, 257, L13 
King, I.R., Stanford, S.A. \& Crane, P. 1995, AJ, 109, 164

Kormendy, J. \& Bender, R. 1999, APJ, 522, 772

Kowalenko, V. \& Melia, F. 1999, MNRAS, 310, 1053

Lauer, T.R., et al. 1993, AJ. 106, 1436

Mcquade, K., Calzetti, D. \& Kinney, A.L. 1995, ApJS, 97, 331

Melia, F. 1992a, ApJ Letters, 387, L25

Melia, F. 1992b, ApJ Letters, 398, L95

Melia, F. 1994, ApJ, 426, 577

Melia, F., Liu, S. \& Coker, R.F. 2000a, ApJ, in press

Melia, F., Liu, S. \& Coker, R.F. 2000b, ApJ Letters, 545, L117

Najarro, F., Krabbe, A., Genzel, R., Lutz, D., Kudritzki, R. P., \& Hillier, D. J. 1997, AA, 325, 700

Narayan, R., Yi, I. \& Mahadevan, R. 1995, Nature, 374, 623

Neugebauer, G., et al. 1984, ApJ Letters, 278, L1

Rossi, P., Bodo, G., Massaglia, S. \& Ferrari, A. 1997, AA, 321, 672

Rubin, V. \& Ford, W.K. Jr. 1971, ApJ, 170, 25

Shapiro, S.L. 1973, APJ, 180, 531

Soifer, B.T., et al. 1986, APJ, 304, 651

Stanek, K.Z. \& Garnavich, P.M. 1998 ApJ Letters, 503, L131

Tremaine, S. 1995, AJ, 110, 628

Trinchieri, G. \& Fabbiano, G. 1991, APJ, 382, 82 
This manuscript was prepared with the AAS IATEX macros v4.0. 


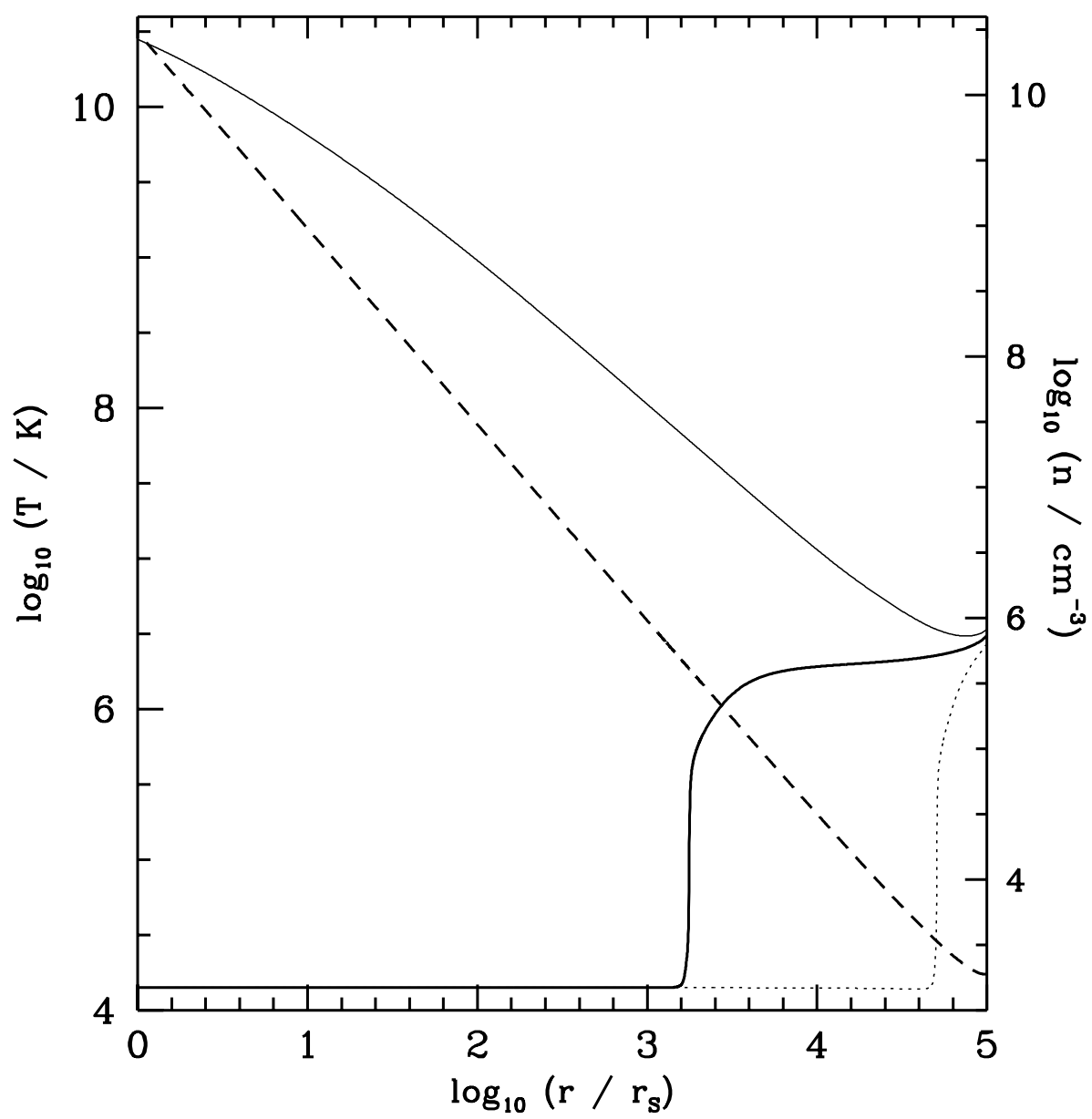

Fig. 1.- Temperature profile for the accreting gas, for three values of the initial temperature at the outer radius $r_{0}=10^{5} r_{S}\left(\approx 0^{\prime \prime} 08\right)$. The accretion rate in all cases is $\dot{M}=1.5 \times 10^{24} \mathrm{~g} \mathrm{~s}^{-1}$. The initial temperature is characterized by the ratio of thermal to gravitational energy density at $r_{0}$, which is 0.19 (thin, solid curve - the hot branch solution), 0.17 (thick, solid curve - the best fit model for M31*) and 0.15 (dotted curve). Also shown here is the run of proton density (dashed curve) of the best fit model, whose scale appears on the right hand side. 


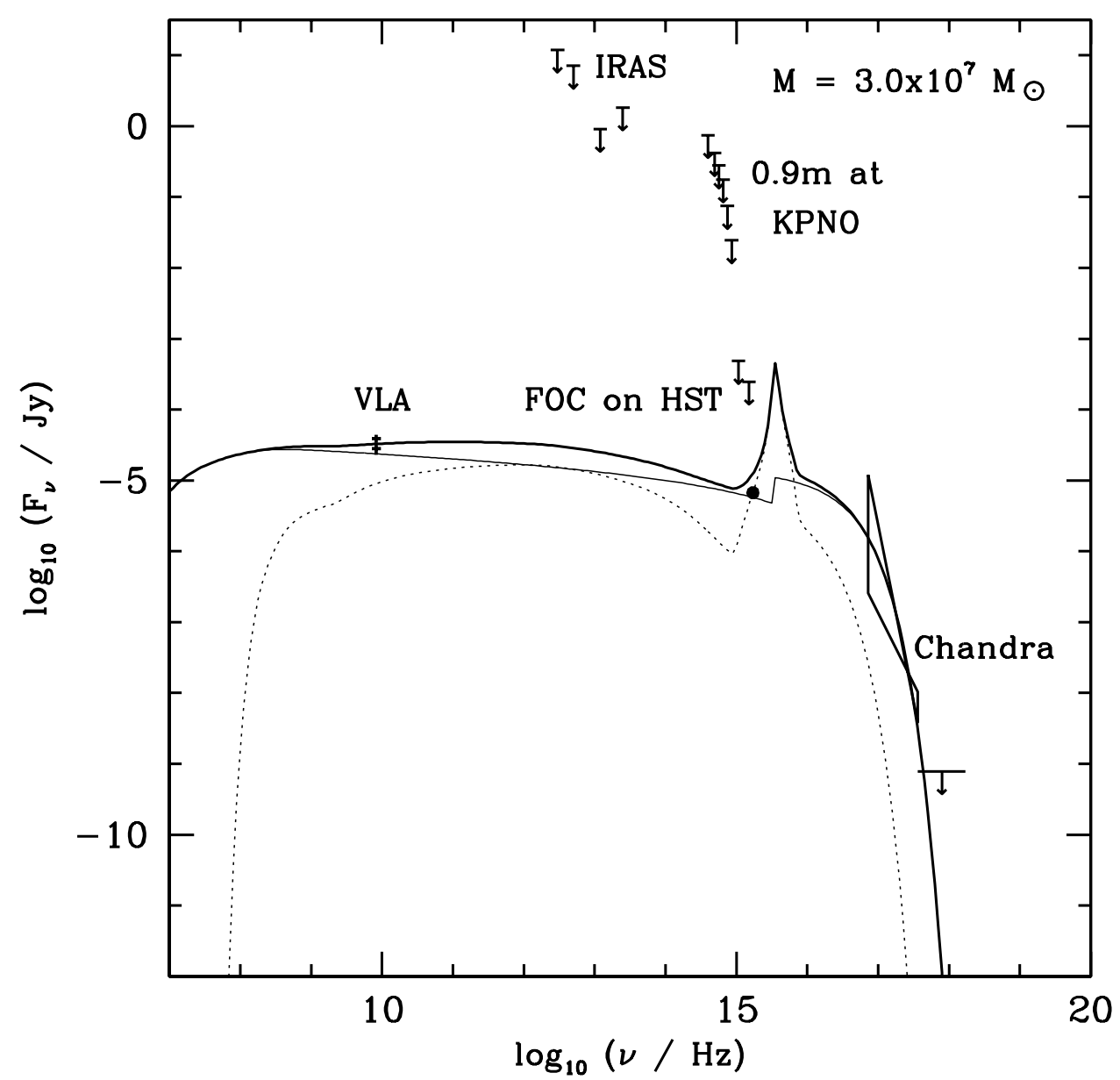

Fig. 2.- Spectrum for the best fit model. The parameter values are specified in the caption of Figure 1. Here, the thin solid curve represents the contribution from the outer hot $\left(T>10^{6} \mathrm{~K}\right)$ region, beyond $\sim 10^{3} r_{S}$. The step in the curve is due to radiative recombination. The dotted curve corresponds to the emissivity from the inner cool region, which accounts for the prominent UV peak due to hydrogen line emission. 